\title{
Differentiation of Compact and Extended Conformations of Di-Ubiquitin Conjugates with Lysine-Specific Isopeptide Linkages by Ion Mobility-Mass Spectrometry
}

\author{
Ji Eun Jung, ${ }^{1}$ Nicholas A. Pierson, ${ }^{2}$ Andreas Marquardt, ${ }^{1}$ Martin Scheffner, ${ }^{1}$ \\ Michael Przybylski, ${ }^{1}$ David E. Clemmer ${ }^{2}$ \\ ${ }^{1}$ Laboratories of Analytical Chemistry and Biochemistry, and Graduate School, Chemical Biology, University of Konstanz, \\ Konstanz, Germany \\ ${ }^{2}$ Department of Chemistry, Indiana University, Bloomington, IN, USA
}

\begin{abstract}
Modification of ubiquitin, a key cellular regulatory polypeptide of 76 amino acids, to polyubiquitin conjugates by lysine-specific isopeptide linkage at one of its seven lysine residues has been recognized as a central pathway determining its biochemical properties and cellular functions. Structural details and differences of distinct lysine-isopeptidyl ubiquitin conjugates that reflect their different functions and reactivities, however, are only partially understood. Ion mobility spectrometry (IMS) combined with mass spectrometry (MS) has recently emerged as a powerful tool for probing conformations and topology involved in protein interactions by an electric fielddriven separation of polypeptide ions through a drift gas. Here we report the conformational characterization and differentiation of Lys63- and Lys48-linked ubiquitin conjugates by IMS-MS. Lys63- and Lys48-linked di-ubiquitin conjugates were prepared by recombinant bacterial expression and by chemical synthesis using a specific chemical ligation strategy, and characterized by high-resolution Fourier transform ion cyclotron resonance mass spectrometry, circular dichroism spectroscopy, and molecular modeling. IMS-MS was found to be an effective tool for the identification of structural differences of ubiquitin complexes in the gas phase. The comparison of collision cross-sections of Lys63- and Lys48-linked di-ubiquitin conjugates showed a more elongated conformation of Lys63-linked di-ubiquitin. In contrast, the Lys48linked di-ubiquitin conjugate showed a more compact conformation. The IMS-MS results are consistent with published structural data and a comparative molecular modeling study of the Lys63- and Lys48-linked conjugates. The results presented here suggest IMS techniques can provide information that complements MS measurements in differentiating higher-order polyubiquitins and other isomeric protein linkages.
\end{abstract}

Key words: Lys63- and Lys48-linked di-ubiquitin, Ion mobility-mass spectrometry, Collision cross sections, high-resolution mass spectrometry, Circular dichroism spectroscopy

Both Ji Eun Jung and Nicholas A. Pierson contributed equally to this work.

Electronic supplementary material The online version of this article (doi:10.1007/s13361-011-0158-0) contains supplementary material, which is available to authorized users.

Correspondence to: David E. Clemmer; e-mail: clemmer@indiana.edu

\section{Introduction}

Tbiquitin (Ub) is a small regulatory protein of 76 amino acids, which is transferred to target proteins in an ATPdependent process and is present in all types of eukaryotic cells $[1,2]$. Ubiquitination, a modification in which single or 
multiple ubiquitin molecules are attached to a substrate protein, serves as a signaling function that controls a wide variety of cellular processes [3]. Modification of ubiquitin chains occurs by formation of an isopeptide bond between the carboxy-terminal Gly76 group and an $\varepsilon$-amino group of a lysine residue of ubiquitin [4]. Ubiquitin possesses seven lysines (Lys6, 11, 27, 29, 33, 48, and 63), all of which may be modified, resulting in ubiquitin conjugates of different lengths and shapes [5]. All seven lysine residues can participate in the formation of Ub-Ub linkages, either sequentially using the same lysine residue for conjugation (homo-poly-ubiquitination), or by utilizing several distinct lysines to connect consecutive Ub moieties (hetero-polyubiquitination) $[6,7]$.

The best understood function of polyubiquitin conjugates is a signal targeting the modified protein to the $26 \mathrm{~S}$ proteasome for degradation [8,9]. However, it has become evident in several recent studies that ubiquitin conjugation may also serve non-proteolytic functions [10]. For example, while modification of substrate proteins with Lys48-linked ubiquitin chains serves as a signal to target modified proteins for proteasome-mediated degradation, Lys63-linked polyubiquitin chains have been associated with non-proteolytic processes $[2,11]$. Crystal structure data, in agreement with NMR studies, showed that Lys48- or Lys63-linked ubiquitin chains may exert closed and extended conformations [12], suggesting that different mechanisms of recognition may be involved in ubiquitin pathways $[13,14]$. In fact, according to one current model the differences in tertiary structures reflect distinct binding properties for interaction with ubiquitinbinding domains (UBDs), thus, different biochemical functions [15]. This notion (different ubiquitin chains adopt different tertiary structures that in turn mediate different functions) is supported by structural information emerging for different types of polyubiquitin chains [16] and for UBDs specifically recognizing isomeric ubiquitin conjugates $[17,18]$; nonetheless, the structural basis of distinguishing Lys-specific isomeric ubiquitin chains is still only partially understood.

High-resolution mass spectrometry has been successfully applied for the direct characterization of polyubiquitin conjugates [19], but conformation-specific methods are required for structural differentiation of specific lysinelinked ubiquitin conjugates. Ion mobility-mass spectrometry (IMS-MS) has recently emerged as an efficient tool for the separation and analysis of polypeptides and polypeptide interactions with distinct conformations, topology, and charge structures. A new IMS-IMS-MS technique has been recently developed and used to examine ubiquitin ions in the gas phase [20]. IMS is a rapid, gas-phase separation technique, as typical drift time measurements occur on the millisecond time-scale. Investigating gas-phase structures of protein ions can lead to an improved understanding of intramolecular forces that play an important role in protein folding [21, 22]. Hence, the separative capacity of IMS is highly complementary to MS analysis. The ability of IMS-
MS to resolve populations of protein conformers has been previously demonstrated [20, 23-27].

The aim of the present study was to explore the conformational differentiation of defined Lys48- and Lys63-linked di-ubiquitin conjugates, which were purified and characterized by gel electrophoresis, mass spectrometry (MS), and circular dichroism spectroscopy. We show that ion mobility-mass spectrometry can be used to detect conformational differences between two specific lysinelinked di-ubiquitin peptides in the gas phase. Studies of conformations of isolated di-ubiquitin ions in the gas phase can help to delineate structural variations in polyubiquitin conjugates with different biochemical functions. Furthermore, IMS-MS is highly suitable to provide information that might provide an understanding of the relationship between gas-phase structure and solution conformation.

\section{Experimental}

\section{Material and Reagents}

The following commercially available reagents were used: acrylamide/bis-acrylamide solution, sodium dodecyl sulfate (SDS), Tris-(hydroxymethyl)-aminomethane (Tris), acetonitrile $(\mathrm{MeCN})$, formic acid and isopropanol were purchased from Roth (Karlsruhe, Germany). N-[tri(hydroxymethyl) methyl]glycine (tricine) was obtained from Merck (Darmstadt, Germany). Tetramethylethylenediamine (TEMED), ammonium persulfate (APS), and Coomassie brilliant blue G250 were purchased from Sigma (St. Louis, MO, USA).

\section{Preparation of K63-Linked and K48-Linked Di-Ubiquitin Conjugates}

K63-linked di-ubiquitin (Ub_1) (Enzo Life Sciences, Lörrach, Germany) was produced in E. Coli in $50 \mathrm{mM}$ Tris-buffer containing $1 \mathrm{mM}$ EDTA, pH 7.6). Lys48-linked di-ubiquitin (Ub_2) was prepared by incubating E1 and Ubc13-Mms2 with equal concentrations of ubiquitin capped at the future proximal chain terminus (Asp77-ubiquitin) and ubiquitin capped at the future distal chain terminus (K48R/K63R-ubiquitin). Diubiquitin was enzymatically synthesized by linking proximal and distal chains. The distal terminus of the resulting dimer is de-blocked by treatment with yeast ubiquitin hydrolase-1 (YUH-1) followed by purification by anion exchange chromatography. The proximal terminal Asp77 was removed by treatment with YUH-1.

K48-linked di-ubiquitin of ubiquitin mutants [K48Cubiquitin; Ub_2] and [D77-ubiquitin] were expressed in BL21 ( $\mathrm{DDE} 3)$ E.coli. Proteins were purified by cation exchange chromatography. Di-ubiquitin was enzymatically synthesized by linking [K48C-ubiquitin] and [D77ubiquitin] in the presence of ubiquitin conjugating enzyme E2-25 K (UbcH1) and human ubiquitin activating enzyme E1 [28]. 
The chemical synthesis of K63-linked di-ubiquitin (Ub_3) by chemoselective thioether ligation was previously described in detail [29]. Briefly, the general approach for the synthesis of specific lysine-linked ubiquitin conjugates consisted of (1), preparation of a linear ubiquitin-"donor" peptide with a C-terminal cysteine residue and (2), its conjugation with a corresponding lysine-specific branched bis-haloacylubiquitin "acceptor" peptide by thioether alkylation (details in reference [29]).

\section{Tris-Tricine Polyacrylamide Gel Electrophoresis}

1D-tricine polyacrylamide gels were prepared using a MiniPROTEAN3 cell gel system (Bio-Rad, München, Germany). Samples were dissolved in a buffer containing $4 \%$ SDS, $25 \%$ glycerol, $50 \mathrm{mM}$ Tris, $0.02 \%$ Coomassie blue, and $6 \mathrm{M}$ urea, $\mathrm{pH}$ 6.8. 1D-Tris-Cl/SDS gel electrophoresis was performed using a Tris-Cl/SDS buffer containing $3 \mathrm{M}$ Tris$\mathrm{Cl}$ and $0.3 \%$ SDS, $\mathrm{pH}$ 8.45. Cathode buffer concentration was $0.1 \mathrm{M}$ Tris, $0.1 \mathrm{M}$ tricine, and $0.1 \%$ SDS, and anode buffers were $0.2 \mathrm{M}$ Tris-Cl, $\mathrm{pH}$ 8.9. Stock solutions prepared for separation gels and stacking gels contained $3 \mathrm{M}$ Tris with $0.3 \%$ SDS, glycerol, $10 \%$ ammonium persulfate and TEMED, $\mathrm{pH} 8.45$ and $1.5 \mathrm{M}$ Tris, $0.4 \%$ SDS, $10 \%$ APS and TEMED, $\mathrm{pH}$ 8.8. After loading on a $15 \%$ separating gel, a saturated aqueous isobutyl alcohol solution was added, the gel polymerized for $1 \mathrm{~h}$, and $10 \mu \mathrm{g}$ of protein sample applied. The electrophoresis was developed for $15 \mathrm{~min}$ at $60 \mathrm{~V}$, and subsequently for $1-2 \mathrm{~h}$ at $100 \mathrm{~V}$ and visualized by Coomassie brilliant blue R-250 staining.

\section{Protein Recovery by Passive Elution}

Proteins were extracted directly from the polyacrylamide gel by treatment with an organic solvent mixture in an ultrasonic bath [30]. After staining with Coomassie Blue, the gel was washed and destained in acetic acid/ethanol/ $\mathrm{H}_{2} \mathrm{O}(1: 3: 6$, vol/ $\mathrm{vol} / \mathrm{vol})$. The protein spots were cut from the gel, lyophilized, and gel pieces incubated for $10-30 \mathrm{~min}$ in $30 \mathrm{~mL}$ of a mixture of formic acid/acetonitrile/isopropanol $/ \mathrm{H}_{2} \mathrm{O}$ $(10: 5: 3: 2, \mathrm{vol} / \mathrm{vol} / \mathrm{vol} / \mathrm{vol})$ at $35{ }^{\circ} \mathrm{C}$ in an ultrasonic bath. Crude samples were desalted using the ZipTip cleanup procedure with ZipTip $\mathrm{C}_{4}$ pipette tips (Millipore, Billerica, MA, USA), and the desalted proteins were diluted to a concentration of approximately $1 \mathrm{pmol} / \mu \mathrm{L}$ for MS analysis.

\section{Mass Spectrometric Characterization of Ubiquitin Polypeptides}

Electrospray ionization (ESI) Fourier transform ion cyclotron resonance (FT-ICR) mass spectrometry was performed with a Bruker Apex II Fourier transform ion cyclotron resonance mass spectrometer (Bruker Daltonic GmbH, Bremen, Germany) equipped with a 7.0 Tesla actively shielded superconducting magnet (Magnex, Oxford, UK), and an APOLLO electrospray ionization source (Bruker Daltonic GmbH). Nano-
ESI FT-ICR mass spectrometry was performed with a modified APOLLO ESI source (Bruker Daltonics) to accommodate a custom-made nano-ESI system [19, 31]. A stable spray was obtained by application of a capillary entrance voltage between -880 and $-1120 \mathrm{~V}$, while the nano-ESI needle was grounded. Calibration was performed externally using fragment ions of angiotensin I (Bachem, Bubendorf, Switzerland) produced by in-source collisioninduced dissociation (CID). Acquisition of spectra was performed with the Bruker Daltonics software XMASS and corresponding programs for mass calculation, calibration and processing. A $10 \mu \mathrm{M}$ solution of peptides in $50 \%$ methanol: $2 \%$ aqueous acetic acid $(1: 1, \mathrm{vol} / \mathrm{vol})$ was used for sample preparation.

ESI-ion trap mass spectra were recorded on an Esquire 3000 + ion trap mass spectrometer (Bruker Daltonics) equipped with a standard ESI source and HyStar 3.1 (Bruker Daltonics) control software. Samples were introduced by direct infusion with a syringe pump at flow rates of 3-5 $\mu \mathrm{L} / \mathrm{min}$, using nitrogen both as nebulizer and drying gas. Ion source voltages were: capillary exit, $-200 \mathrm{~V}$; Skimmer, $40 \mathrm{~V}$; Scanned mass range, $m / z$ 200 3000. Samples were desalted using the ZipTip procedure (Millipore, Billerica, MA, USA) and reconstituted in $0.1 \%$ formic acid before injection.

\section{Circular Dichroism Spectroscopy}

CD spectra were recorded with a Jasco model J-720 spectropolarimeter at $25{ }^{\circ} \mathrm{C}$ in $0.05 \mathrm{~cm}$ quartz cells under constant nitrogen flush. The instrument was calibrated with $0.06 \%(\mathrm{wt} / \mathrm{vol})$ ammonium-d-camphor-10-sulfonate (Katayama Chemical, Osaka, Japan) in doubly-distilled water; the sample concentrations were $0.5 \mu \mathrm{g} / \mu \mathrm{L}$. The spectra were averages of six scans between 190 and $260 \mathrm{~nm}$, respectively.

\section{Ion Mobility Spectrometry}

Theoretical and experimental considerations associated with the use of ion mobility spectrometry techniques as a means of assessing information about protein structure have been reviewed previously [23, 32-36]. Nested ion mobility/timeof-flight mass spectrometry measurements were carried out using a home-built instrument at Indiana University. While a number of instrumental designs have been described [37, 38] (and are likely to be valuable for these types of studies), the instrument used for these studies is a low-pressure drift tube that utilizes a drift region created by a series of insulating and conducting spacers which, when compressed, make the cavity that contains the buffer gas.

Ions from $\sim 60 \mu \mathrm{M}$ di-ubiquitin (K63- or K48-) in $99.8 \%$ water: $0.2 \%$ formic acid ( $\mathrm{vol} / \mathrm{vol})$ are produced by nanoscale ESI performed with a TriVersa NanoMate chip-based ESI system (Advion BioSciences, Ithaca, NY, USA) (1.8$2.1 \mathrm{kV}$ bias). The continuous beam of ions that is produced is trapped in a Smith-geometry hourglass ion funnel [39, 
40]. Periodically ( $\sim 60 \mathrm{~ms})$, the trapped ions are introduced as a short pulse $(150 \mu \mathrm{s})$ into the drift region. The drift region in these studies is $1.8 \mathrm{~m}$ in length and operated using a drift field of $10 \mathrm{~V} \cdot \mathrm{cm}^{-1}$ and a buffer gas pressure of 3.0 Torr of $\mathrm{He}$ at $300 \mathrm{~K}$. Ions exit the drift region through a differentially-pumped interface and are extracted into a high-vacuum region $\left(5 \times 10^{-7}\right.$ Torr $)$, and focused into the source region of a reflectron-geometry time-of-flight mass spectrometer.

Collision cross-sections are determined from measured drift times according to Eq. (1):

$$
\Omega=\frac{(18)^{1 / 2}}{16} \frac{z e}{\left(k_{b} T\right)^{1 / 2}}\left[\frac{1}{m_{I}}+\frac{1}{m_{B}}\right]^{1 / 2} \frac{t_{d} E}{L} \frac{760}{P} \frac{T}{273.2} \frac{1}{N},
$$

where $z e, k_{b}, m_{l}$, and $m_{B}$ are the ion charge, Boltzmann's constant, the mass of the ion, and the mass of the helium buffer gas, respectively; $t_{d}, E$, and $L$ correspond to the ion drift time, the electric field strength, and the drift length, respectively; $P, T$, and $N$ are the pressure, temperature, and neutral number density of the buffer gas, respectively.

\section{Molecular Modeling}

The Hyperchem 7.01 (HyperCube Inc., FL, USA) software was used for the molecular modeling calculations. The initial model was constructed from the crystal structure of wildtype ubiquitin [41]. Two of these structures were fitted together to give a di-ubiquitin molecule. Geometry optimizations (in vacuo) were performed using the Amber99 parameter set for the force field and the Polak-Ribiere algorithm with an RMS gradient of $0.1 \mathrm{kcal} /\left(\AA^{*} \mathrm{~mol}\right)$. Excess charges were not considered in these calculations.

\section{Results and Discussion}

\section{High-Resolution Mass Spectrometric Structural Characterization of Lysine-Specific Di-Ubiquitin Conjugates}

Lys63- and Lys48-linked di-ubiquitin conjugates (Ub_1Ub_3) were prepared by recombinant expression in E. coli cells, and by chemical synthesis using selective thioether ligation as described in the Experimental section [29]. For specific enzymatic ligation of Lys63- and Lys48-chains by an isopeptide bond to the $\varepsilon$-amino group of the distal carboxy-terminal Gly76 group, the Lys48- and Lys63residues of the distal ubiquitin were replaced by Arg (Lys48Arg and Lys63Arg), respectively, in order to avoid oligomerization (see sequences of di-ubiquitin conjugates in Scheme 1). The crude sample of the recombinant Lys63linked di-ubiquitin (Ub_1) containing several byproducts was dissolved in Tris/EDTA buffer, lyophilized, and purified on a $15 \%$ Tris-Tricine SDS gel, showing a major band at $\sim 17.1 \mathrm{kDa}$ (Figure S1, available in Supplementary Material). For mass spectrometric analysis, the excised band was purified by

\section{Ub_1. Recombinant K63-linked di-ubiquitin}

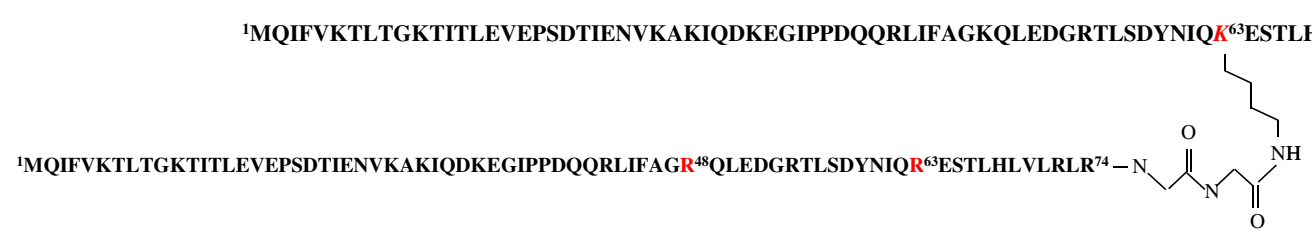

\section{Ub_2. Recombinant K48-linked di-ubiquitin}

${ }^{1}$ MQIFVKTLTGKTITLEVEPSDTIENVKAKIQDKEGIPPDQQRLIFAGC ${ }^{48}$ QLEDGRTLSDYNIQKESTLHLVLRLRGG $^{76}$

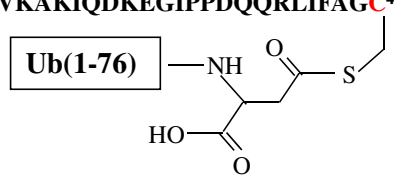

\section{Ub_3. Synthetic K63-linked di-ubiquitin}

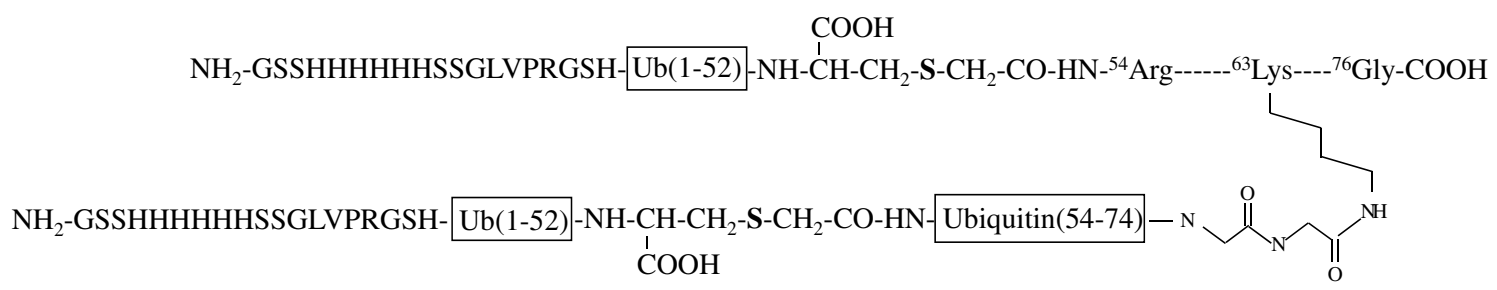

Scheme 1. Primary structures of recombinant and chemically synthesized di-ubiquitin conjugates Ub_1, Ub_2, and Ub_3 
passive elution, which effectively extracted the di-ubiquitin conjugates into solution [30, 42]. Determination of the exact monoisotopic masses by FT-ICR MS confirmed the correct sequences of recombinant Lys-linked di-ubiquitin conjugates for the conjugate $\mathbf{U b} \mathbf{1}$ (Table 1). The experimental values of the ions observed in the mass spectrum matched the predicted abundant charge values, $11+(\mathrm{m} / \mathrm{z} 1561.64), 12+(\mathrm{m} / \mathrm{z}$ $1431.56), 13+(m / z 1321.52)$, and $14+(m / z 1227.23)$ yielding an average-isotopic molecular weight of $17167.53 \mathrm{Da}$ $\left(\mathrm{C}_{756} \mathrm{H}_{1256} \mathrm{~N}_{214} \mathrm{O}_{235} \mathrm{~S}_{2}\right)$. The mass accuracy between calculated and experimental monoisotopic mass obtained by FTICR MS was approximately 3 ppm (Table 1 ).

Recombinant Lys48-linked di-ubiquitin was prepared according to the analogous procedure as described above, by enzymatic linkage of the proximal ubiquitin mutant [K48C] and a distal ubiquitin [D77] with the ubiquitin conjugating enzyme E2-25 $\mathrm{K}(\mathrm{UbcH} 1)$ and ubiquitin activating enzyme E1. A clearly distinguishable band corresponding to K48-linked di-ubiquitin was detected with a molecular weight of $\sim 17.2 \mathrm{kDa}$, and excised from the gel and isolated by passive elution. The molecular mass of the Cys48- (instead of K48) linked di-ubiquitin (Ub_2) was determined by ESI-ion trap-MS and showed a series yielding an average isotope molecular weight of $17201.56 \mathrm{Da}$, consistent with the correct sequence and the elemental formula, $\mathrm{C}_{757} \mathrm{H}_{1254} \mathrm{~N}_{210} \mathrm{O}_{238} \mathrm{~S}_{3}$. The nano-ESI FT-ICR mass spectrum showed a single homogeneous peptide product with the experimental molecular mass 17191.20 Da, consistent with the calculated monoisotopic molecular mass (17191.16 Da; mass accuracy 1.75 ppm) (Table 1).

\section{Conformational Characterization by $C D$ Spectroscopy}

The conformational preferences of the di-ubiquitin conjugates in water were analyzed by CD spectroscopy (Fig. 1). The comparative $\mathrm{CD}$ spectrum of bovine ubiquitin monomer in water showed a negative band at $208 \mathrm{~nm}\left(\pi-\pi^{*}\right.$ transition) and a small negative shoulder around $226 \mathrm{~nm}$, characteristic of an unordered structure. The Lys-linked di-ubiquitin conjugates did not indicate significant conformational differences. The spectra of the Lys63-linked di-ubiquitin (Ub_1)

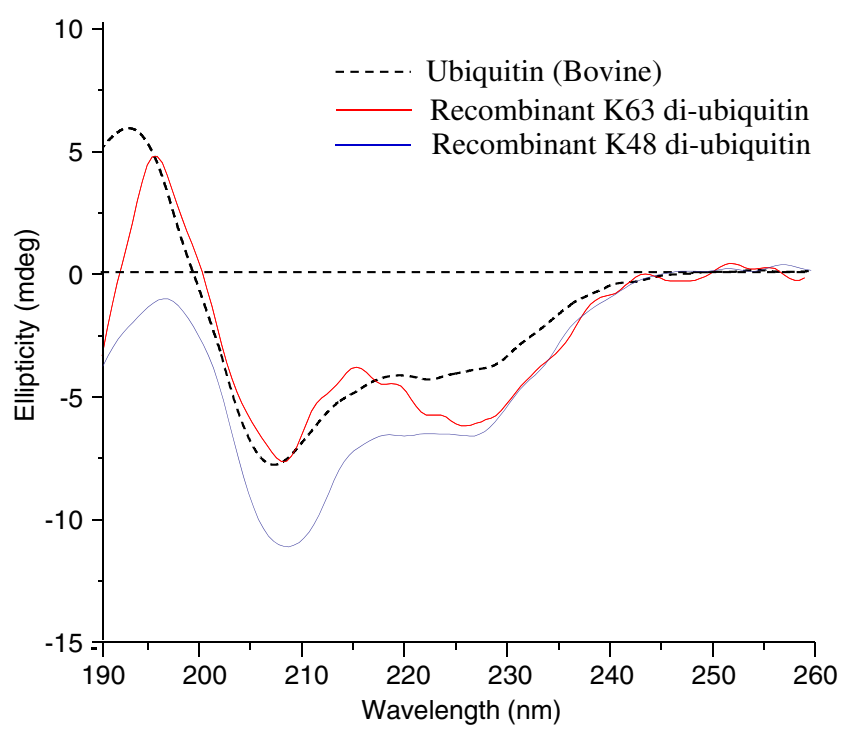

Figure 1. CD spectra of di-ubiquitin conjugates with Lys63and Lys48-specific linkages

in water showed the presence of a negative band around $208 \mathrm{~nm}$ and an additional strongly negative band around $227 \mathrm{~nm}$, indicative of an $\alpha$-helical structure. The CD spectrum of the Lys48- (Ub_2) in comparison to the Lys63-linked di-ubiquitin showed a slight shift in the $\pi-\pi^{*}$ electronic transitions towards higher wavelengths and the presence of a negative shoulder at approximately $227 \mathrm{~nm}$ $\left(\mathrm{n}-\pi^{*}\right.$ transition). These results indicated similar conformational preferences for the di-ubiquitin conjugates in aqueous solution, with Ub_1 having slightly higher random coil content than $\mathbf{U b} \_\mathbf{1}$.

\section{Comparison of Collision Cross-Sections and Conformational Characterization by Ion Mobility Spectrometry-Mass Spectrometry}

The conformations of different charge states of monomeric forms of ubiquitin and other small model protein ions produced by ESI have been discussed in some detail $[20,34,43-45]$. A few studies have reported information about dimeric and other early stage aggregation forms of

Table 1. Mass Spectrometric Characterization of Lys63- and Lys48-linked Di-Ubiquitin Conjugates

\begin{tabular}{|c|c|c|c|c|}
\hline & \multirow[t]{2}{*}{ Peptide } & \multicolumn{3}{|c|}{ ESI-ion trap $\mathrm{MS}^{\mathrm{a}} /$ Nano-ESI-FTICR-MS ${ }^{\mathrm{b}}$} \\
\hline & & $\mathrm{MW}_{\text {calc }}$ & $\mathrm{MW}_{\text {found }}{ }^{\mathrm{c}}$ & Mass accuracy $[\mathrm{ppm}$ \\
\hline \multirow[t]{2}{*}{ Ub_1 } & Recombinant & 17167.53 & $17167.77^{\mathrm{a}}$ & $14^{\mathrm{a}}$ \\
\hline & K63-linked di-ubiquitin & 17157.25 & $17157.39^{\mathrm{b}}$ & $3.2^{\mathrm{b}}$ \\
\hline \multirow[t]{2}{*}{ Ub_2 } & Recombinant & 17201.56 & $17201.97^{\mathrm{a}}$ & $12.20^{\mathrm{a}}$ \\
\hline & K48-linked di-ubiquitin & 17191.16 & $17191.20^{\mathrm{b}}$ & $1.75^{\mathrm{b}}$ \\
\hline Ub_3 & Synthetic K63-linked di-ubiquitin conjugate & 21383.97 & $21384.77^{\mathrm{a}}$ & $1.4^{\mathrm{a}}$ \\
\hline
\end{tabular}

${ }^{a}$ Average molecular mass; ESI-ion trap mass spectra were recorded on an Esquire 3000+ ion trap mass spectrometer (Bruker Daltonics, Bremen, Germany) equipped with a standard ESI source.

${ }^{\mathrm{b}}$ Monoisotopic mass; nano-ESI mass spectrometric analyses were performed with a Bruker APEX II FT-ICR instrument equipped with an actively shielded $7 \mathrm{~T}$ superconducting magnet, and an APOLLO electrospray ionization source (Bruker Daltonics).

${ }^{\mathrm{c}}$ The most abundant isotopic peak was selected for calculation of MW. 
peptides and proteins [46-49]. Here, we focus on comparative studies of the Lys63-linked (Ub_1) and Lys48-linked (Ub_2) ubiquitin conjugates. Nested $t_{\mathrm{d}}(\mathrm{m} / \mathrm{z})$ datasets [50] for these two systems are shown in Fig. 2. The charge state envelope for the Lys63-linked diubiquitin conjugate (Fig. 2a) is centered around a slightly lower $m / z$ than that of Lys48-linked di-ubiquitin (Fig. 2b); six common charge states $\left([\mathrm{M}+14 \mathrm{H}]^{14+}-[\mathrm{M}+19 \mathrm{H}]^{19+}\right)$ are observed and labeled in Fig. 2.

For the Lys63-linked di-ubiquitin, the charge states $[\mathrm{M}+$ $13 \mathrm{H}]^{13+}$ through $[\mathrm{M}+18 \mathrm{H}]^{18+}$ appear as two predominant types of conformational states, one partially folded (higher mobility/shorter drift time) and one elongated (lower mobility/longer drift time). As we have noted previously for the ubiquitin monomer [43], these relatively broad peaks
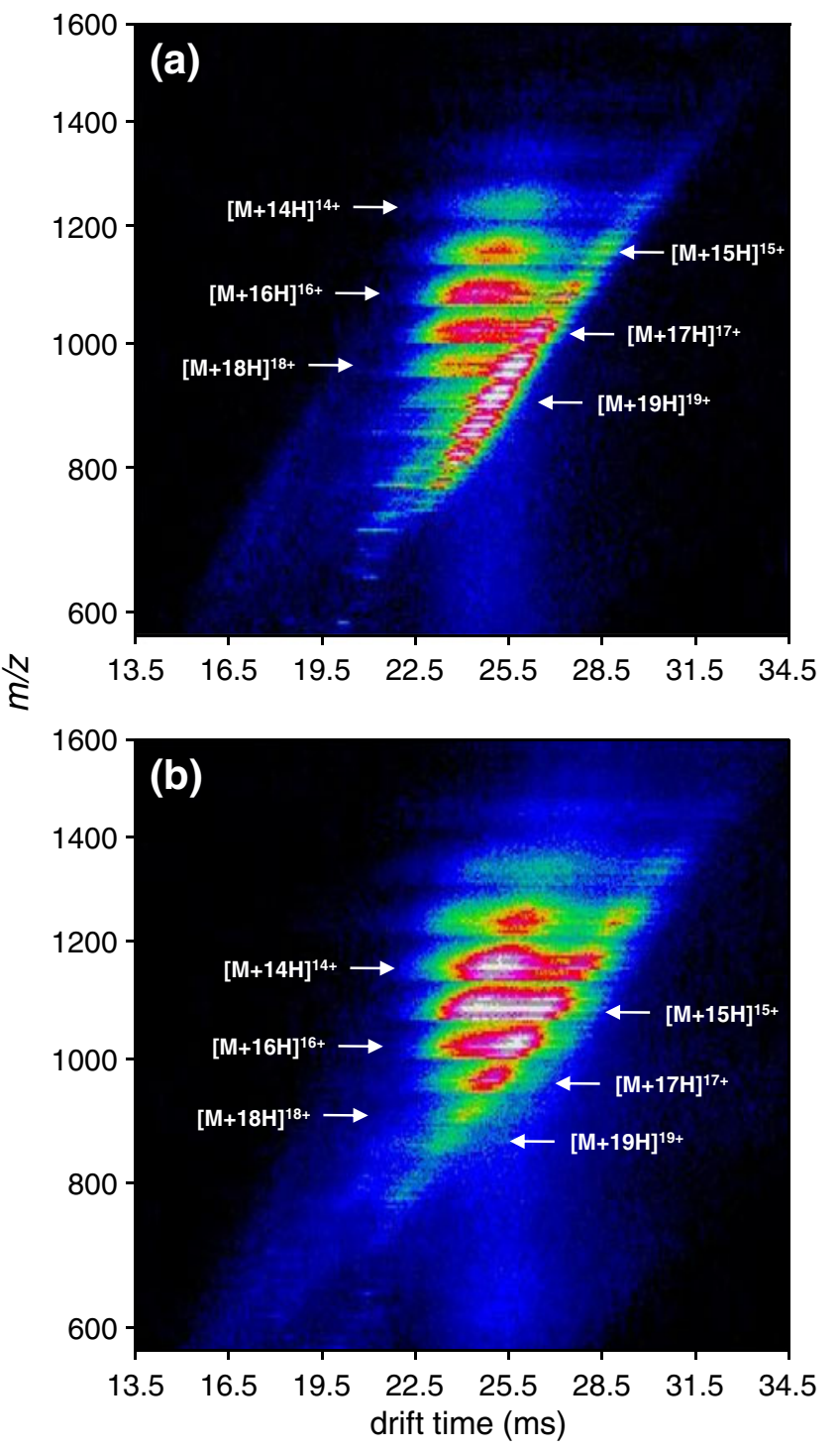

Figure 2. Nested $t_{\mathrm{d}}(\mathrm{m} / \mathrm{z})$ plots for ubiquitin conjugates $\mathrm{K} 63$ di-Ub (Ub_1) (a) and K48 di-Ub (Ub_2) (b). Two-dimensional features are displayed as a false-color scale, which reflects ion abundance. $[\mathrm{M}+14 \mathrm{H}]^{14+}$ to $[\mathrm{M}+19 \mathrm{H}]^{19+}$ charge states are labeled for each protein might be composed of many sub-populations of states, having similar cross sections that are not separated in the present studies. Partially-folded and elongated conformations are also observed for the Lys48-linked di-ubiquitin charge states $[\mathrm{M}+11 \mathrm{H}]^{11+}$ through $[\mathrm{M}+16 \mathrm{H}]^{16+}$. Elongated conformations persisted for higher charge states, $[\mathrm{M}+17 \mathrm{H}]$ ${ }^{17+}-[\mathrm{M}+19 \mathrm{H}]^{19+}$ and $[\mathrm{M}+19 \mathrm{H}]^{19+}-[\mathrm{M}+22 \mathrm{H}]^{22+}$ for $\mathrm{K} 48$ di-Ub and K63 di-Ub, respectively.

Drift time distributions are converted to collision crosssection distributions according to Eq. (1) described in the Experimental section, and are shown in Fig. 3. Partiallyfolded conformations possess smaller cross sections (2500-3100 $\AA^{2}$ ), while elongated conformations have larger cross sections $\left(3100-3830 \AA^{2}\right)$. Cross sections for partially-folded conformations were identical for each respective charge state of Lys63- and Lys48-linked diubiquitin. For elongated conformers, however, the Lys63linked conjugate exhibited larger cross sections (for all charge states) compared to those of the Lys48-linked conjugate (Table 2). Differences in the collision crosssections of the elongated conformations of the di-ubiquitin conjugates range from $2.1 \%$ for the $14+$ charge state to $3.7 \%$ for the $18+$ charge state.

On average, the elongated conformations of Lys63-linked Ub_1 are approximately 3\% larger than conformers corresponding to the Lys48-linked Ub_2. With an instrumental resolving power of $\sim 50$ to $10 \overline{0}$, such differences are relatively easy to observe. In the present system, peaks are substantially broader than the instrumental capabilities. This arises when multiple structures are not resolved, or when transitions occur as ions migrate through the drift tube [25, 43]. In the present system, the peak shapes and positions are relatively different, allowing the K63- and K48-linked diubiquitin conjugates to be differentiated. It is important to note that a mixture of the conjugates would be fairly difficult to resolve, even though the techniques have relatively high resolving power. The observed differences in the mobilities of the di-ubiquitin conjugates suggest that screening of higher-order polyubiquitins and proteins with different isomeric linkages by IMS-MS can complement MS as a means of characterizing structure.

\section{Comparative Molecular Modeling of Di-Ubiquitin Conjugates}

Comparative structural models of the recombinant Lys63linked di-ubiquitin conjugate Ub_1, recombinant Lys48linked di-ubiquitin conjugate Ub_2, and the chemically synthesized Lys63-linked di-ubiquitin Ub_3, obtained by molecular modeling using the Hyperchem-7 software, are shown in Fig. 4. The overall structures calculated for the conjugates suggest conformational stabilities with spatial separation of both parts of the Lys48- and Lys-63 branching, respectively, in agreement with previously reported X-ray crystal structures and with NMR structures [12, 13]. Consistent with the ion mobility spectrometry data, the 


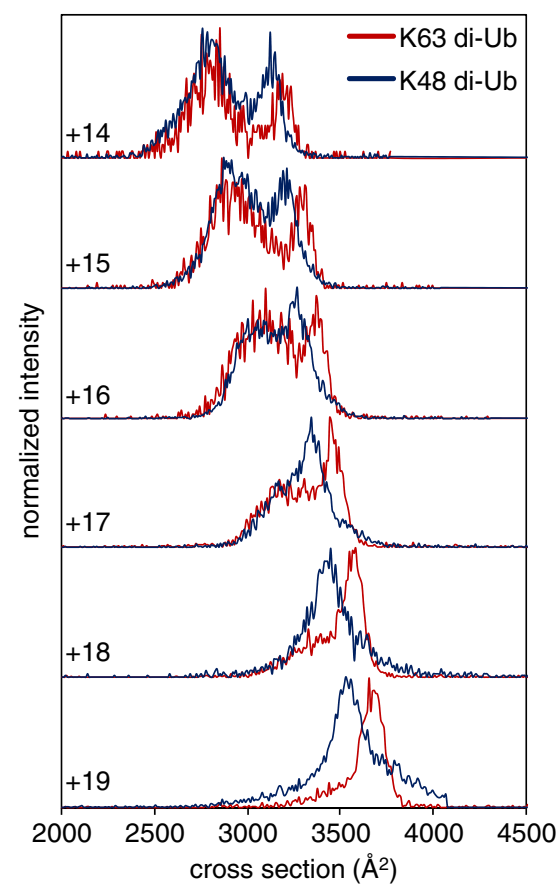

Figure 3. Collision cross-section distributions of the common charge states between K63 di-Ub and K48 di-Ub. Drift time distributions of each charge state are produced by taking mass spectral slices of the 2D data set shown in Fig. 2; collision cross-section distributions are then generated from ion drift times as outlined in the Experimental section. Intensities of all distributions are normalized to unity

structure models indicate a considerably more extended conformation of the Lys63-linked di-ubiquitin. No significant differences were observed between the recombinant Lys63-linked conjugate containing mutated Lys residues and the chemically synthesized conjugate $\mathbf{U b} \_\mathbf{3}$, containing a thioether linkage in the proximal ubiquitin chain.

It is interesting to compare the calculated collision crosssections of the K63- and K48-linked di-ubiquitin conjugates Ub_1 and Ub_2 shown in Fig. 4. Using the MOBCAL suite of programs, we determined projection [51], exact hard spheres scattering [52], and trajectory [53, 54] model cross sections. The most accurate of these calculations comes from the trajectory model, which yields values of $\Omega(\mathrm{calc})=1930$ and $1806 \AA^{2}$ for $\mathbf{U b} \_\mathbf{1}$ and $\mathbf{U b} \mathbf{2}$, respectively. These values are far below the experimental collision cross-section range

Table 2. Ion Mobility Data of Elongated Conformers of the Di-Ubiquitin Conjugates

\begin{tabular}{lccc}
\hline Charge state & \multicolumn{2}{c}{$\operatorname{ccs}\left(\AA^{2}\right)$} & \% Difference \\
\cline { 2 - 3 } & K63 di-Ub & K48 di-Ub & \\
\hline 14 & 3186 & 3120 & 2.1 \\
15 & 3308 & 3209 & 3.0 \\
16 & 3370 & 3264 & 3.2 \\
17 & 3444 & 3339 & 3.1 \\
18 & 3570 & 3442 & 3.7 \\
19 & 3670 & 3544 & 3.5 \\
\hline
\end{tabular}
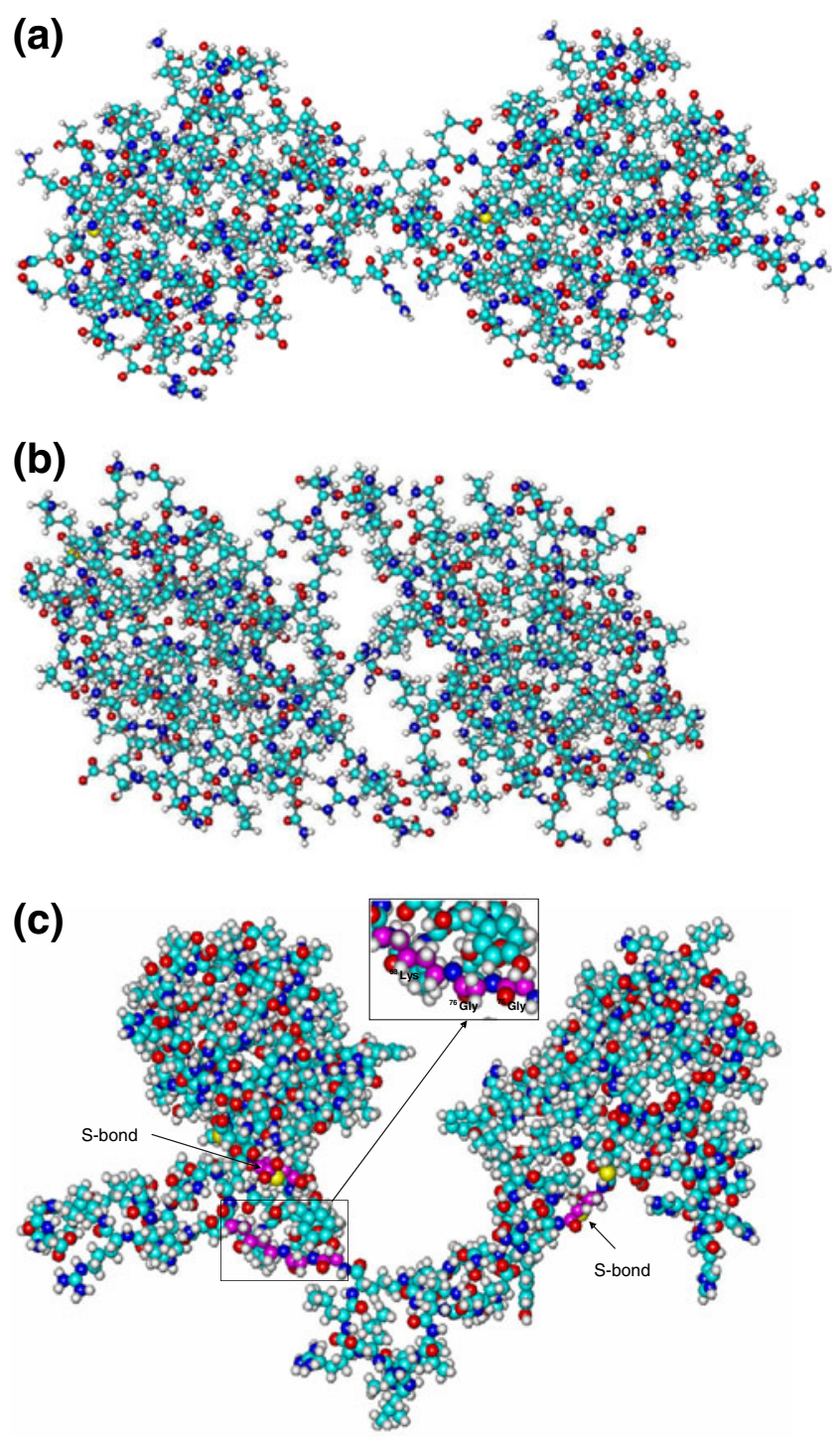

Figure 4. Structural model of the recombinant Lys63-linked di-ubiquitin conjugate Ub_1 (a), the Lys48-linked di-ubiquitin conjugate Ub_2 (b), and synthesized Lys63-linked di-ubiquitin Ub_3 (c). The Lys63 linkage in (c) is highlighted in the insert

$\left[\Omega(\operatorname{expt})=2500-3830 \AA^{2}\right]$ that we measured. This difference arises because we have not included the effect of Coulomb repulsion (arising from multiple charging) in these modeled systems. The geometries reported here are calculated from energy minimized, gas-phase models of $\mathbf{U b} \mathbf{1}$ and $\mathbf{U b} \mathbf{2}$ constructed from the condensed-phased structure of the monomer, and we did not attempt to sample the many conformations of the protonated gas-phase ions. This said, experiment and theory are in agreement with regards to the relative ordering of the sizes for the K63- and K48-linked di-ubiquitin conjugates.

\section{Conclusions}

We have presented a combined application of ion mobilitymass spectrometry together with high-resolution MS, CD 
spectroscopy, and molecular modeling, for the characterization of the conformations of Lys63- and Lys48-linked di-ubiquitin conjugates. The results indicate that in marked contrast to Lys48-linked chains, Lys63-linked chains are considerably more elongated than Lys48-linked di-ubiquitin. Cross-section distributions appear identical for compact structures that exist for the +13 to +17 charge states of both conjugates, while elongated conformers showed distinct differences for the specific lysine- $\varepsilon$-amino linkages. Elongated conformers exist for both types of conjugates in the cross-section distributions of charge states +14 to +19 , with collision cross-sections for these ions ranging from $\sim 3100 \AA^{2}$ for $[\mathrm{K} 48 \mathrm{di}-\mathrm{Ub}+14 \mathrm{H}]^{14+}$ to $\sim 3700 \AA^{2}$ for $[\mathrm{K} 63 \mathrm{di}-\mathrm{Ub}+19 \mathrm{H}]^{19+}$. For the elongated conformers, collision cross-sections of K63-linked di-ubiquitin are on average $3 \%$ larger than those of K48-linked di-ubiquitin, indicating that K48-linked conjugates adopt a more compact structure than K63-linked conjugates.

These structural results for the gas-phase ions are consistent with previous NMR spectroscopy data in solution. Characterizing the structures of proteins in the absence of solvent is suggested to be useful for (1), the comparison of gas-phase results with structural information from solution provides insight about how solvent interactions influence structure; (2), the characterization of gas-phase ion structures may become an important factor in the development of new analytical techniques for studying mixtures of protein conformers.

\section{Acknowledgments}

The authors acknowledge support for this work by grants from the Deutsche Forschungsgemeinschaft (DFG), Bonn, Germany and the Graduate Research School Chemical Biology of the University of Konstanz; and by an Indiana University grant from the National Institutes of Health (1RC1GM090797-01), as well as funding through the DoD NSWC Crane "Next Generation Threat Detection" (N00164-08-C-JQ11). The expert assistance by Dr. Reinhold Weber with the highresolution spectrometry is gratefully acknowledged.

\section{References}

1. Weissman, A.: Themes and variations on ubiquitylation. Nat Rev Mol Cell Biol 2, 169-178 (2001)

2. Pickart, C.M., Eddins, M.J.: Ubiquitin: Structures, functions, mechanisms. Biochim Biophys Acta 1695, 55-72 (2004)

3. Pickart, C.M.: Mechanisms underlying ubiquitylation. Annu Rev Biochem 70, 503-533 (2001)

4. Fang, S., Weissman, A.M.: A field guide to ubiquitylation. Cell Mol Life Sci 61, 1546-1561 (2004)

5. Peng, J., Schwartz, D., Elias, J.E., Thoreen, C.C., Cheng, D., Marsischky, G., Roelofs, J., Finley, D., Gygi, S.P.: A proteomics approach to understanding protein ubiquitination. Nat Biotechnol 21, 921-926 (2003)

6. Pickart, C.M., Fushman, D.: Polyubiquitin chains: Polymeric protein signals. Curr Opin Chem Biol 8, 610-616 (2004)

7. Li, W., Ye, Y.: Polyubiquitin chains: Functions, structures, and mechanisms. Cell Mol Life Sci 65, 2397-2406 (2008)

8. Ciechanover, A.: The ubiquitin-proteasome pathway: On protein death and cell life. EMBO J 17(24), 7151-7160 (1998)
9. Glickman, M.H., Ciechanover, A.: The ubiquitin-proteasome proteolytic pathway: Destruction for the sake of construction. Physiol Rev 82, 373-428 (2002)

10. Strous, G.J., Govers, R.: The ubiquitin-proteasome system and endocytosis. J Cell Sci 112, 1417-1423 (1999)

11. Hershko, A., Ciechanover, A.: The ubiquitin system. Annu Rev Biochem 67, 425-479 (1998)

12. Tenno, T., Fujiwara, K., Tochio, H., Iwai, K., Morita, E.H., Hayashi, H., Murata, S., Hiroaki, H., Sato, M., Tanaka, K., Shirakawa, M.: Structural basis for distinct roles of Lys63- and Lys48-linked polyubiquitin chains. Genes Cells 9, 865-875 (2004)

13. Hicke, L., Schubert, H.L., Hill, C.P.: Ubiquitin-binding domains. Nat Rev Mol Cell Biol 6, 610-621 (2005)

14. Hofmann, K., Bucher, P.: The UBA domain: A sequence motif present in multiple enzyme classes of the ubiquitination pathway. Trends Biochem Sci 21, 172-173 (1996)

15. Ikeda, F., Dikic, I.: Atypical ubiquitin chains: New molecular signals. protein modifications: beyond the usual suspects review series. EMBO Rep 9, 536-542 (2008)

16. Phillips, C.L., Thrower, J., Pickart, C.M., Hill, C.P.: Structure of a new crystal form of tetraubiquitin. Acta Cryst D57, 341-344 (2001)

17. Ryabov, Y.E., Fushman, D.: A model of interdomain mobility in a multidomain protein. J Am Chem Soc 129, 3315-3327 (2007)

18. Ohno, A., Jee, J., Fujiwara, K., Tenno, T., Goda, N., Tochio, H., Kobayashi, H., Hiroaki, H., Shirakawa, M.: Structure of the UBA domain of Dsk2p in complex with ubiquitin: molecular determinants for ubiquitin recognition. Structure 13, 521-532 (2005)

19. Przybylski, M., Glocker, M.O.: Electrospray mass spectrometry of biomacromolecular complexes with noncovalent interactions-New analytical perspectives for supramolecular chemistry and molecular recognition processes. Angew Chem 35, 806-826 (2001)

20. Koeniger, S.L., Merenbloom, S.I., Valentine, S.J., Jarrold, M.F., Udseth, H.R., Smith, R.D., Clemmer, D.E.: An IMS-IMS analogue of MS-MS. Anal Chem 78, 4161-4174 (2006)

21. Onuchic, J.N., Luthey, S.Z., Wolynes, P.G.: Theory of protein folding: The energy landscape perspective. Annu Rev Phys Chem 48, 545-600 (1997)

22. Jarrold, M.F.: Peptides and proteins in the vapor phase. Ann Rev Phys Chem 51, 179-207 (2000)

23. Bohrer, B.C., Merenbloom, S.I., Koeniger, S.L., Hilderbrand, A.E., Clemmer, D.E.: Biomolecule analysis by ion mobility spectrometry. Annu Rev Anal Chem 1(10), 1-10 (2008)

24. Shelimov, K.B., Jarrold, M.F.: Conformations, unfolding, and refolding of apomyoglobin in vacuum: An activation barrier for gas-phase protein folding. J Am Chem Soc 119, 2987-2994 (1997)

25. Koeniger, S.L., Clemmer, D.E.: Resolution and structural transitions of elongated states of ubiquitin. J Am Soc Mass Spectrom 18, 322-331 (2007)

26. Plasencia, M.D., Isailovic, D., Merenbloom, S.I., Mechref, Y., Clemmer, D.E.: Resolving and assigning N-linked glycan structural isomers from ovalbumin by IMS-MS. J Am Soc Mass Spectrom 19, 1706-1715 (2008)

27. Barnes, C.A.S., Hilderbrand, A.E., Valentine, S.J., Clemmer, D.E.: Resolving isomeric peptide mixtures: A combined HPLC/ion mobilityTOFMS analysis of a 4000-component combinatorial library. Anal Chem 74, 26-36 (2002)

28. Chae, Y.K., Lee, H., Lee, W.: Ubiquitin fusion system for recombinant peptide expression and purification: Application to the cytoplasmic domain of syndecan-4. Bull Korean Chem Soc 28, 1549-1552 (2007)

29. Jung, J., Wollscheid, H.P., Marquardt, A., Manea, M., Scheffner, M., Przybylski, M.: Functional ubiquitin conjugates with lysine- $\varepsilon$-aminospecific linkage by thioether ligation of cysteinyl-ubiquitin peptide building blocks. Bioconj Chem 20, 1152-1162 (2009)

30. Ehring, H., Strömberg, S., Tjernberg, A., Norén, B.: Matrix-assisted laser desorption/ionization mass spectrometry of proteins extracted directly from sodium dodecyl sulphate-polyacrylamide gels. Rapid Commun Mass Spectrom 11, 1867-1873 (1997)

31. Damoc, E., Youhnovski, N., Crettaz, D., Tissot, J.D., Przybylski, M.: High resolution Pproteome analysis of cryoglobulins using fourier transform-ion cyclotron resonance mass spectrometry. Proteomics 3, 1425-1433 (2003)

32. Liu, Y., Valentine, S.J., Counterman, A.E., Hoaglund, C.S., Clemmer, D.E.: Injected-ion mobility analysis of biomolecules. Anal Chem 69, 728A-735A (1997) 
33. Clemmer, D.E., Jarrold, M.F.: Ion mobility measurements and their applications to clusters and biomolecules. J Mass Spectrom 32, 577592 (1997)

34. Hoaglund Hyzer, C.S., Counterman, A.E., Clemmer, D.E.: Anhydrous protein ions. Chem Rev 99, 3037-3079 (1999)

35. Jarrold, M.F.: Unfolding, refolding, and hydration of proteins in the gas phase. Acc Chem Res 32, 360-367 (1999)

36. Wyttenbach, T., Bowers, M.T.: Gas-phase conformations: The ion mobility/ion chromatography method. Top Curr Chem 225, 207-232 (2003)

37. Hoaglund-Hyzer, C.S., Clemmer, D.E.: Ion trap/ion mobility/quadrupole/ time-of-flight mass spectrometry for peptide mixture analysis. Anal Chem 73, 177-184 (2001)

38. Hoaglund-Hyzer, C.S., Lee, Y.J., Counterman, A.E., Clemmer, D.E.: Coupling ion mobility separations, collisional activation techniques, and multiple stages of MS for analysis of complex peptide mixtures. Anal Chem 74, 992-1006 (2002)

39. Shaffer, S.A., Tang, K.Q., Anderson, G.A., Prior, D.C., Udseth, H.R., Smith, R.D.: A novel ion funnel for focusing ions at elevated pressure using electrospray ionization mass spectrometry. Rapid Commun Mass Spectrom 11, 1813-1817 (1997)

40. Shaffer, S.A., Prior, D.C., Anderson, G.A., Udseth, H.R., Smith, R.D.: An ion funnel interface for improved ion focusing and sensitivity using electrospray ionization mass spectrometry. Anal Chem 70, 4111-4119 (1998)

41. Vijay-Kumar, S., Bugg, C.E., Cook, W.J.: Structure of ubiquitin refined at 1.8 a resolution. $J$ Mol Biol 194, 531-544 (1987)

42. Cohen, S.L., Chait, B.T.: Mass spectrometry of whole proteins eluted from sodium dodecyl sulfate-polyacrylamide gel electrophoresis gels. Anal Biochem 247, 257-267 (1997)

43. Koeniger, S.L., Merenbloom, S.I., Clemmer, D.E.: Evidence for many resolvable structures within conformation types of electrosprayed ubiquitin ions. J Phys Chem B 110, 7017-7021 (2006)

44. Li, J., Taraszka, J.A., Counterman, A.E., Clemmer, D.E.: Influence of solvent composition and capillary temperature on the conformations of electrosprayed ions: Unfolding of compact ubiquitin conformers from pseudonative and denatured solutions. Int J Mass Spectrom 185/186/ 187, 37-47 (1999)

45. Badman, E., Myung, S., Clemmer, D.E.: Evidence for unfolding and refolding of gas phase cyctrochrome $c$ ions in a paul trap. J Am Soc Mass Spectrom 16, 1493-1497 (2005)

46. Counterman, A.E., Valentine, S.J., Srebalus, C.A., Henderson, S.C., Hoaglund, C.S., Clemmer, D.E.: High-order structure and dissociation of gaseous peptide aggregates that are hidden in mass spectra. $J \mathrm{Am} \mathrm{Soc}$ Mass Spectrom 9, 743-759 (1998)

47. Barran, P.E., Polfer, N.C., Campopiano, D.J., Clarke, D.J., LangridgeSmith, P.R.R., Langley, R.J., Govan, J.R.W., Maxwell, A., Dorin, J.R., Millar, R.P., Bowers, M.T.: Is it biologically relevant to measure the structures of small peptides in the gas-phase? Int J Mass Spectrom 240, 273-284 (2005)

48. Bernstein, S.L., Dupuis, N.F., Lazo, N.D., Wyttenbach, T., Condron, M.M., Bitan, G., Teplow, D.B., Shea, J.E., Ruotolo, B.T., Robinson, C. V., Bowers, M.T.: Amyloid- $\beta$ protein oligomerization and the importance of tetramers and dodecamers in the etiology of Alzheimer's disease. Nat Chem 1, 326-331 (2009)

49. Ashcroft, A.E.: Mass spectrometry and the amyloid problem-How far can we go in the gas phase? J Am Soc Mass Spectrom 21, 1087-1096 (2010)

50. Hoaglund, C.S., Valentine, S.J., Sporleder, C.R., Reilly, J.P., Clemmer, D.E.: Three-dimensional ion mobility/TOFMS analysis of electrosprayed biomolecules. Anal Chem 70, 2236-2242 (1998)

51. von Helden, G., Hsu, M.T., Gotts, N., Bowers, M.T.: Carbon cluster cations with up to 84 atoms: Structures, formation mechanism, and reactivity. J Phys Chem 97, 8182-8192 (1993)

52. Shvartsburg, A.A., Jarrold, M.F.: An exact hard-spheres scattering model for the mobilities of polyatomic ions. Chem Phys Lett 261, 86-91 (1996)

53. Mesleh, M.F., Hunter, J.M., Shvartsburg, A.A., Schatz, G.C., Jarrold, M.F.: Structural information from ion mobility measurements: Effects of the long-range potential. J Phys Chem 100, 16082-16086 (1996)

54. Shvartsburg, A.A., Schatz, G.C., Jarrold, M.F.: Mobilities of carbon cluster ions: Critical importance of the molecular attractive potential. $J$ Chem Phys 108, 2416-2423 (1998) 